\title{
Bilateral giant extragastrointestinal stromal tumour of the retroperitoneum
}

\author{
Milena Potic Floranovic ${ }^{1}$, Jablan Stankovic ${ }^{2}$, Filip Velickovic ${ }^{3}$, Ana Ristic Petrovic ${ }^{4}$, Ljubinka Jankovic Velickovic ${ }^{4}$ \\ ${ }^{1}$ Scientific Research Centre for Biomedicine, Medical Faculty, University of Niš, Niš, Serbia \\ 2Urology Clinic - Clinical Centre of Niš, Niš, Serbia \\ ${ }^{3}$ Nuclear Medicine Centre - Clinical Centre of Niš, Niš, Serbia \\ ${ }^{4}$ Pathology and Pathological Anatomy Centre - Clinical Centre of Niš, Niš, Serbia
}

Gastroenterology Rev 2019; 14 (3): 217-220

DOI: https://doi.org/10.5114/pg.2019.84730

Address for correspondence: Milena Potic Floranovic, Scientific Research Centre for Biomedicine, Medical Faculty, University of Niš, Niš, Serbia, e-mail: milenapotic@yahoo.com

Gastrointestinal stromal tumours (GISTs) are mesenchymal neoplasms that can appear in any segment of the gastrointestinal (Gl) tract and account for $0.1-3 \%$ of all Gl tumours. They can be benign and malignant, and there are three histological types: spindle, epithelial, and mixed-cell [1]. Almost all GISTs express immunopositivity for CD117, a C-Kit proto-oncogene protein, which shows an increase in the function of C-Kit gene, which encodes growth factor receptor with tyrosine kinase activity [2]. Extragastrointestinal stromal tumours (EGISTs) are extremely rare neoplasms with histopathological and immunohistochemical features similar to GIST. However, they are found outside the Gl tract, in the omentum, retroperitoneum, or mesentery with no evidence of a primary Gl neoplasm, and without connection to the intestinal wall [3]. Less than 60 cases of EGIST localised in retroperitoneum are currently documented in English literature [4].

We present a case of giant bilateral retroperitoneal EGIST in a 58-year-old male patient.

A 58-year-old obese male presented with a sixmonth history of abdominal discomfort. Ultrasound depicted hydronephrosis grade II in the right kidney and large tumour mass (around $20 \mathrm{~cm}$ ) in the projection of the left kidney. Magnetic resonance imaging (MRI) depicted two tumour masses: one adjacent to the left kidney measuring $20 \times 18 \times 25 \mathrm{~cm}$, which filled Gerota's fascia and suppressed nearby structures in the opposite direction; and another, paracaval mass on the right side measuring $50 \times 39 \times 35 \mathrm{~mm}$, compressing the right ureter causing grade II hydronephrosis, which required percutaneous nephrostomy. Renal vasography showed that the tumour mass in the left retroperitoneum did not originate from the kidney, and extirpation of this giant tumour was performed.
Grossly, the tumour measured $22 \times 20 \times 16 \mathrm{~cm}$ and weighed $2 \mathrm{~kg}$ (Figure 1). It was lobulated with adipose tissue on the surface. The cut surface revealed a fish flesh appearance with focusses of degeneration and haemorrhage. Tissue was fixed in $4 \%$ neutral formaldehyde, embedded in paraffin, cut into 4- $\mu$ m sections, and stained with haematoxylin-eosin. An immunohistochemical analysis was performed and included: CD117, vimentin, actin, Anti-MYO D1, CD34, MelanA, S-100, CD68, CD57, CD31, LCA, and CKAE1/AE3.

Histologically, the tumour was mostly hypercellular, composed of short and fusiform spindle cells, with light cytoplasm and elongated nuclei (Figure 2). They were arranged in short less defined fascicles. The tumour appeared well circumscribed; however, infiltration to the adjacent adipose tissue was detected (Figure 3). Parts of the tumour showed interstitial hyalinisation and cells with epithelioid morphology, degenerative nuclear atypia, and multinucleation. Numerous mitotic figures and giant bizarre cells were also noted (Figure 4). Immunohistochemical analysis showed strong and diffuse positivity for CD117 and vimentin (Figures 5, 6), while the other applied markers were negative. The proliferation index was $45 \%$. All clinical and radiological investigations, including microscopic features and immunohistochemical profile of tumour cells, supported an EGIST diagnosis - a subtype of spindle cell sarcoma. One month after postoperative recovery, in another surgical procedure, a second, smaller paracaval tumour mass was removed, which intraoperatively presented as lymph nodes. Grossly, the second tumour measured $75 \times 60 \times 60 \mathrm{~mm}$ and showed identical histological features as the tumour in the left retroperitoneum, with no evidence of any lymph node structure (Figure 7). 


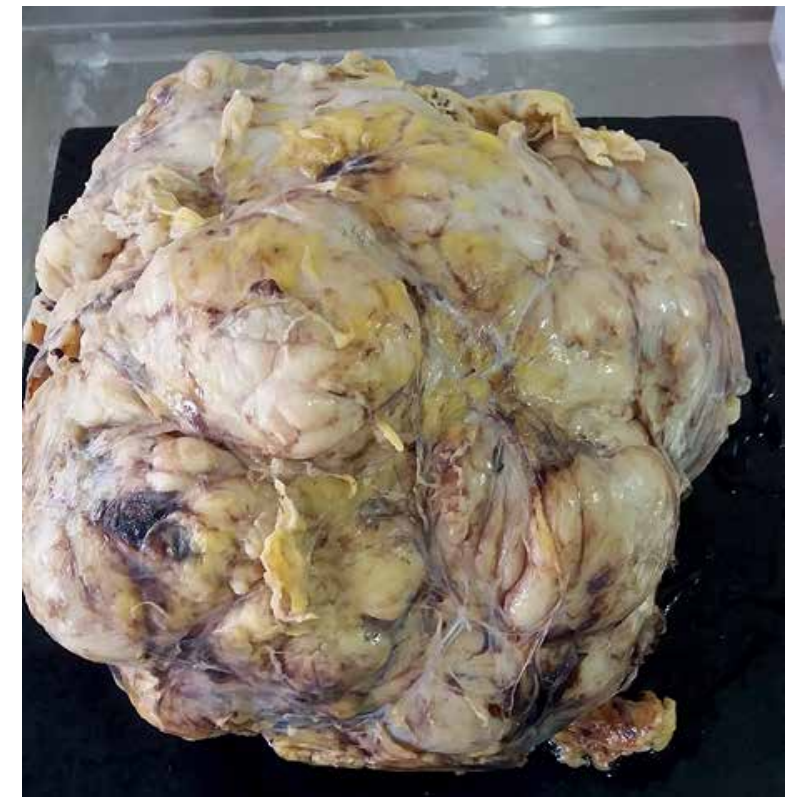

Figure 1. Macroscopic appearance of EGIST

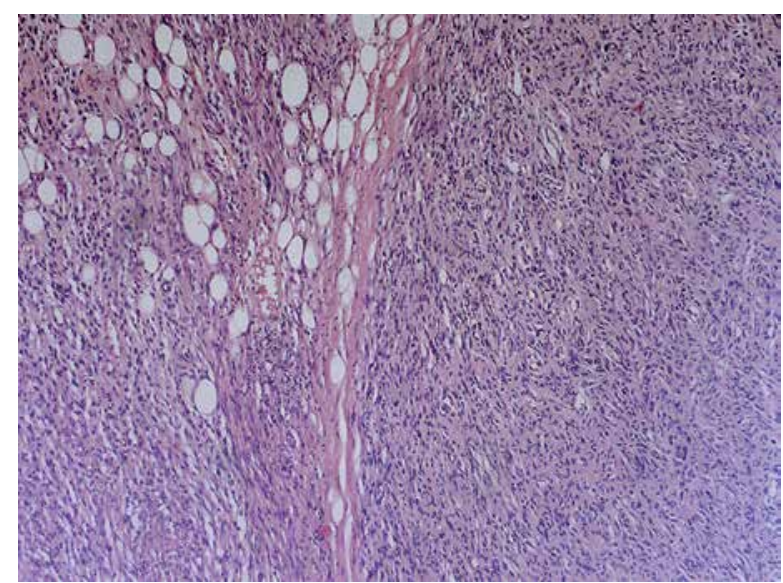

Figure 3. Tumour infiltrating adjacent adipose tissue $(H \& E$ 10x)

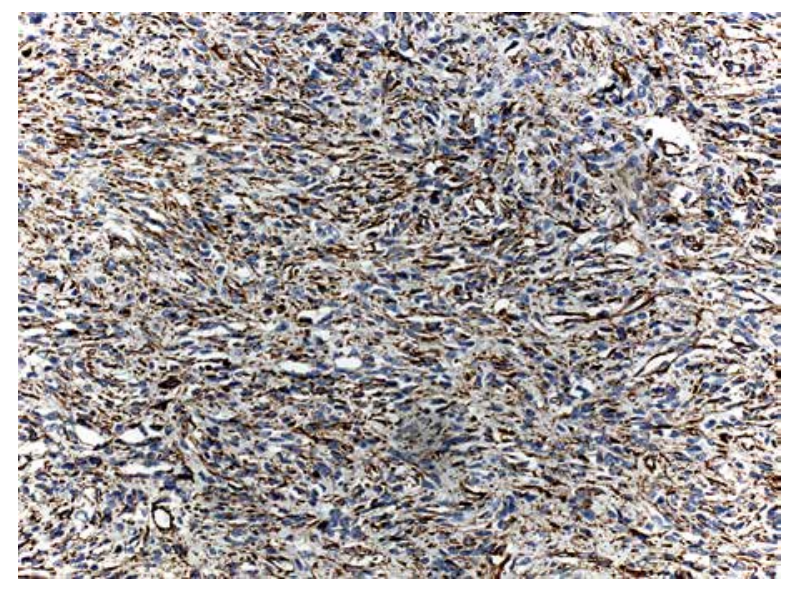

Figure 5. Strong, diffuse positivity for vimentin

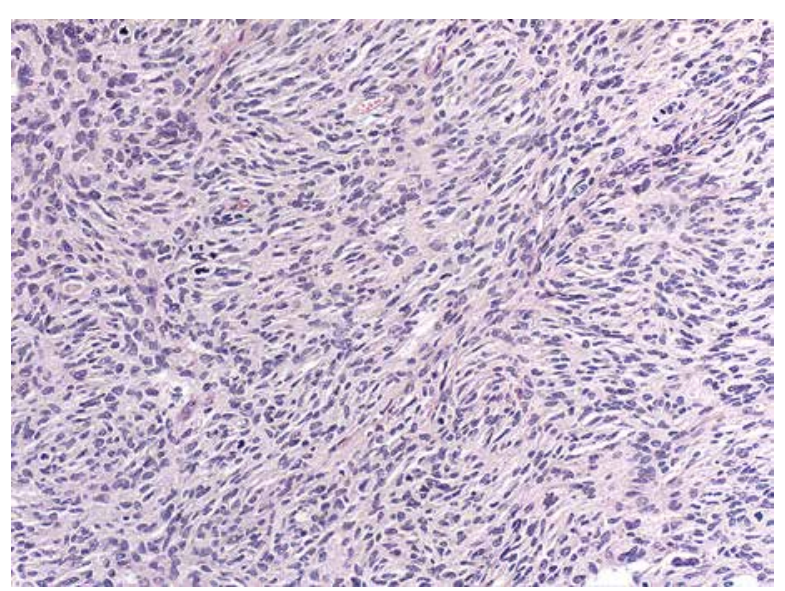

Figure 2. Short, elongated cells arranged in less defined fascicles (H\&E 20x)

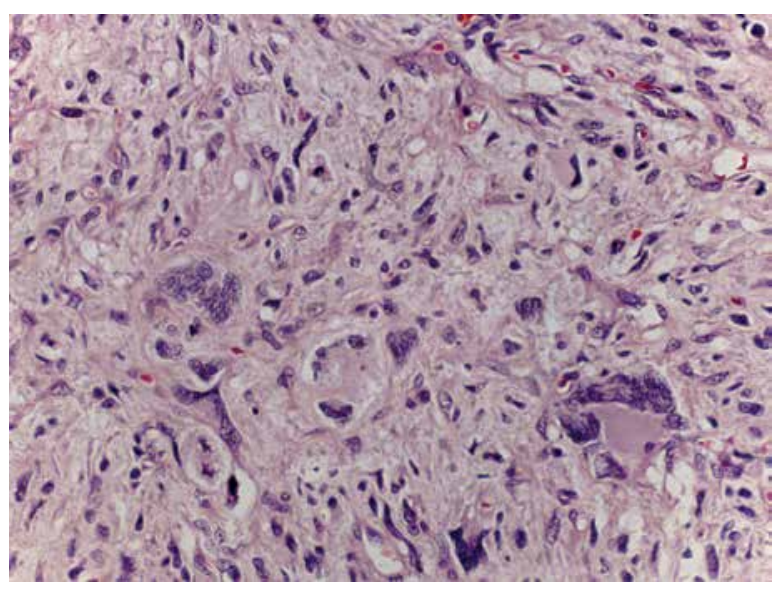

Figure 4. Bizarre multinucleated giant cells (H\&E 40x)

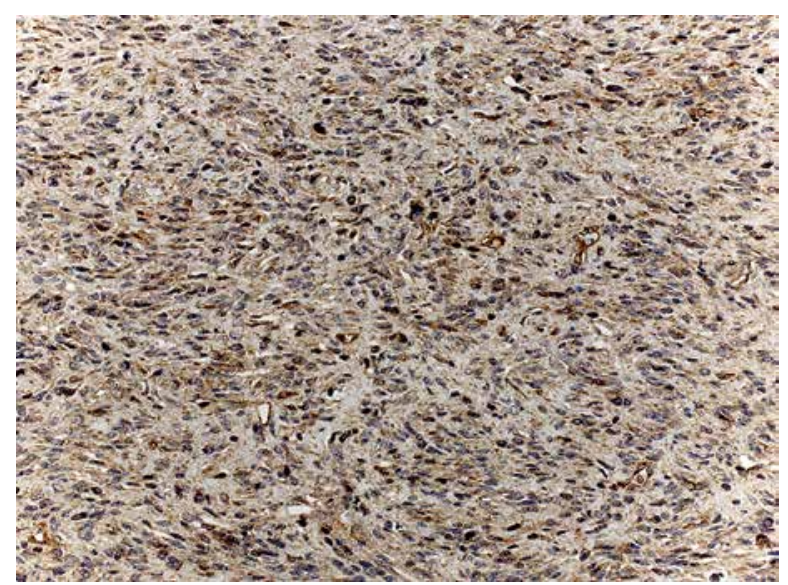

Figure 6. Strong, diffuse positivity for CD117 
This case illustrates one of the largest reported EGISTs in the retroperitoneum. EGISTs are morphologically, immunohistochemically, and molecularly similar to GISTs. They are rare, accounting for less than $5 \%$ of GISTs [3]. They usually appear in literature as case reports with a few multiple case studies [5-7]. About $80 \%$ of EGISTs are located in the omentum, mesentery, or retroperitoneum. Cases in different sites of the $a b$ domen, such as pancreas, spleen, mediastinum, and in the pelvis as vulvovaginal/rectovaginal septal masses, have been described [8]. Their hallmark is complete absence of a connection with the GI wall. A large study by Agaimy and Wunsh reported that most cases of supposed EGIST arose from the outermost layer of the external muscularis propria of the gut wall or appeared to be due to metastasis from the primary GIST. In our case, the diagnostic workups, such as CT and endoscopies, proved that both tumours were genuine EGISTs.

Due to the absence of mucosal involvement, EGISTs are often asymptomatic, in contrast to GISTs, which can present with various symptoms: haematemesis, melena, palpable mass, abdominal pain, vomiting, weight loss, abdominal distention, or intestinal obstruction [3]. Hence, the diagnosis of EGISTS is delayed, and they tend to grow larger than GISTs before causing any obstructive symptoms. In our case, the patient had not lost weight and, apart from abdominal discomfort, showed no other symptoms. His obesity masked the presence of the tumour, enabling its growth. That led to the development of the tumour to its enormous size and compression on the contralateral structures.

The behaviour of GISTs differs by localisation. The majority of GISTs located in the stomach have good prognosis, while those in the small intestine have significantly worse prognosis $[5,6]$. EGISTs usually behave more aggressively. Factors that indicate poor prognosis include tumour size greater than $5 \mathrm{~cm}$, mitotic rate greater than 1-5/10 HPFs, presence of tumour necrosis or metastasis, and most recently the c-kit mutation [5-7]. In our patient, both giant retroperitoneal tumours were detected on the first MRI, and the smaller tumour at the right side developed simultaneously with the larger tumour, with continuous growth between the two surgical procedures.

In $95 \%$ of cases, GISTs show immunoreactivity for the CD117 marker C-Kit tyrosine kinase receptor. GISTs are also positive on vimentin, CD34 (40-70\%), and smooth muscle actin, but they do not stain for desmin and S-100 [9, 10]. CD117, CD34, and vimentin are normally expressed on the interstitial cells of Cajal pacemaker cells, present throughout the wall of normal adult GI tract. That leads to the presumption that GISTs orig-

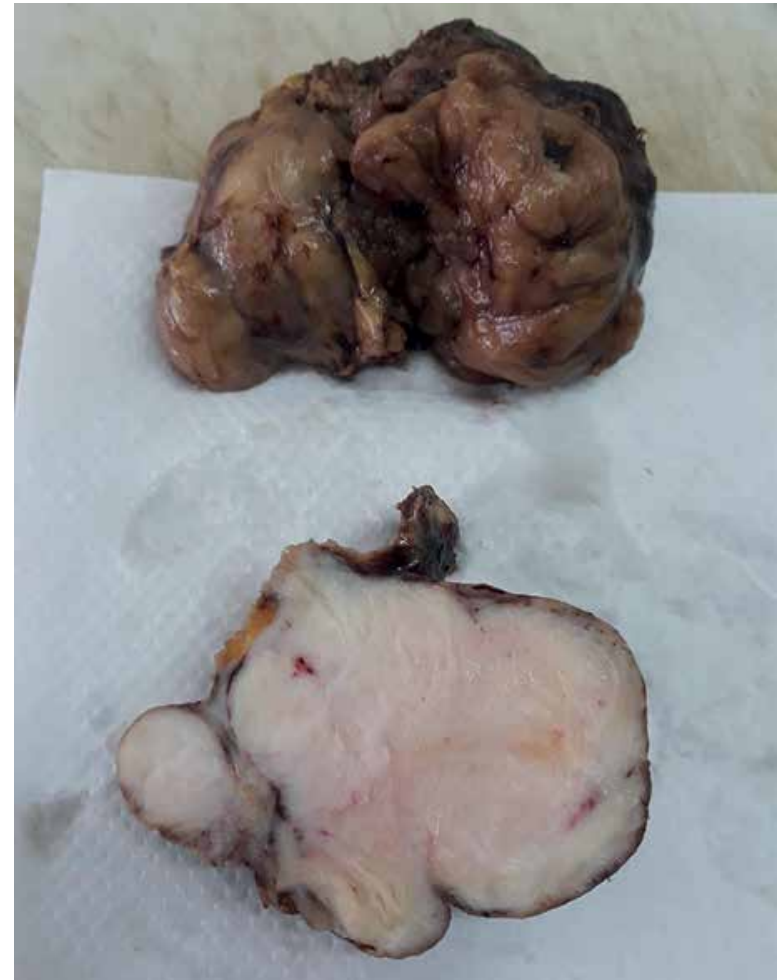

Figure 7. Macroscopic features of second paracaval tumour

inate from Cajal cells. The origin of EGISTs, however, remains controversial. Some theories suggest that EGISTs develop through extensive extramural growth of GISTs, which leads to the disappearance of the connection with the GI wall [7]. Others suggest that GISTs arise from a common precursor cell of the interstitial cells of Cajal and smooth muscle, enabling them to develop inside and outside the GI tract [2, 3]. Retroperitoneal EGIST, like typical GIST, shows positive staining for CD117. Staining for other immunological markers is variable: B-cell lymphoma 2 (BCL-2) (80\%), CD34 (70\%), smooth muscle actin (35\%), S100 (10\%), and desmin (5\%) [4]. Our case showed strong positivity to CD117 and vimentin. CD117 is the most selective immunohistochemical marker differentiating GISTs from smooth muscle tumours [5, 7, 9]. Our case was CD34 negative, which is a less specific feature of GISTs. CD117 expression can be detected in other neoplasms such as: melanoma, dermatofibrosarcoma protuberans, seminoma, liposarcoma, malignant fibrous histiocytoma, Ewing's sarcoma, myofibrosarcoma, haemangiopericytoma, fibrosarcoma, synovial sarcoma, and others [11]. Differential diagnosis of abdominal or pelvic mass with other tumours requires a panel of antibodies. Since our case was negative for MelanA, S-100, Actin, AntimyoD1, and other noted markers, those diagnoses were ruled out [12]. 
Complete surgical resection is the best treatment for GISTs. Imatinib mesylate, which is an inhibitor of the tyrosine kinase activity of c-kit, has proven to be an effective and safe adjuvant postoperative therapy for GISTS, and useful for controlling metastatic GIST disease as well [13]. However, the role of imatinib in the treatment of EGISTs is still unclear.

CD117-positive stromal tumours, although rare, can involve the retroperitoneum. The presence of bilateral giant retroperitoneal tumour with CD117 expression, should be considered as an EGIST if there is no connection to the gastrointestinal tract. The management of these tumours demands their surgical removal because they are chemoresistant. Because of their rarity, further studies are needed to clearly define the phenotypic and genetic characteristics as well as the biological behaviour of these tumours.

\section{Acknowledgments}

This work was supported by a grant No. 175092 from the Ministry of Education and Science of the Republic of Serbia.

\section{Conflict of interest}

The authors declare no conflict of interest.

\section{References}

1. Miyahira, CK, Bonfitto M, de Lima Farto JF, et al. Extragastrointestinal stromal tumor: a differential diagnosis of compressive upper abdominal tumor. Case Rep Surg 2018; 2018: 1052960.

2. Vij M, Agrawal V, Pandey R. Malignant extra-gastrointestinal stromal tumor of the pancreas. a case report and review of literature. J Pancreas 2011; 12: 200-4.

3. Priya V, Kumari N, Krishnani N. Extraintestinal gastrointestinal stromal tumor: is it biologically different from gastrointestinal stromal tumor. Gastrointest Cancer Res Ther 2017; 2: 1015.

4. Watal P, Brahmbhatt SG, Thoriya PJ, Bahri NU. Retroperitoneal extragastrointestinal stromal tumor: radiologic pathologic correlation. J Clin Imaging Sci 2014; 4: 34.

5. Reith JD, Goldblum JR, Lyles RH, Weiss SW. Extragastrointestinal (soft tissue) stromal tumors: an analysis of 48 cases with emphasis on histologic predictors of outcome. Mod Pathol 2000; 13: 577-85.

6. Hatipoğlu E. Extragastrointestinal stromal tumor (EGIST): a 16year experience of 13 cases diagnosed at a single center. Med Sci Monit 2018; 24: 3301-6.

7. Agaimy A, Wunsh PH. Gastrointestinal stromal tumours: a regular origin in the muscularis propria, but an extremely diverse gross presentation. A review of 200 cases to critically re-evaluate the concept of so-called extra-gastrointestinal stromal tumours. Langenbecks Arch Surg 2006; 39: 322-9.

8. Antonopoulos P, Leonardou P, Barbagiannis N, et al. Gastrointestinal and extragastrointestinal stromal tuomrs: report of two cases and review of the literature. Case Rep Gastroenterol 2014; 8: 61-6.
9. Güler B, Özyılmaz F, Tokuç B, et al. Histopathological features of gastrointestinal stromal tumors and the contribution of DOG1 expression to the diagnosis. Balkan Med J 2015; 32: 388-96.

10. Skandalos IK, Hotzoglou NF, Matsi KC, et al. Giant extra gastrointestinal stromal tumor of lesser omentum obscuring the diagnosis of a choloperitoneum. Int J Surg Case Rep 2013; 4: 818-21.

11. Matteo D, Dandolu V, Lembert L, et al. Unusually large extraintestinal GIST presenting as an abdomino-pelvic tumor. Arch Gynecol Obstet 2008; 278: 89-92.

12. Hong X, Choi H, Loyer EM, et al. Gastrointestinal stromal tumor: role of $\mathrm{CT}$ in diagnosis and in response evaluation and surveillance after treatment with imatinib. Radiographics 2006; 26: 481-95.

13. Blanke CD, Demetri GD, von Mehren M, et al. Long-term results from a randomized phase II trial of standard-versus higher-dose imatinib mesylate for patients with unresectable or metastatic gastrointestinal stromal tumors expressing KIT. J Clin Oncol 2008; 26: 620-5.

Received: 19.11 .2018

Accepted: 31.01 .2019 